\section{2/2013 bereits zum dritten Mal lanciert}

Nunmehr zum 3. Mal wird der renommierte CAMLOG Foundation-Forschungspreis der CAMLOG Foundation ausgeschrieben. Der Forschungspreis wird alle 2 Jahre anlässlich des Internationalen CAMLOG Kongresses vergeben und steht allen jungen Wissenschaftlern sowie Fachleuten aus Universität, Klinik und Praxis im Alter bis 40 Jahren offen.

Die erwarteten außergewöhnlichen wissenschaftlichen Arbeiten, die in englischer oder deutscher Sprache verfasst sein können, müssen in einem anerkannten Fachjournal publiziert werden und eines der folgenden Themen aus der dentalen Implantologie oder verwandten Disziplinen behandeln:

- Diagnostik und Planung in der dentalen Implantologie

- Hart- und Weichgewebe-Management

- Nachhaltigkeit implantatgestützter Prothetik
- physiologische und pathophysiologische Aspekte in der dentalen Implantologie

- Fortschritte digitaler Verfahren in der dentalen Implantologie.

Die Beiträge werden vom CAMLOG Foundation Board beurteilt und bewertet. Der Gewinner des CAMLOG Foundation-Forschungspreises 2012 / 2013 erhält anlässlich des Internationalen CAMLOG Kongresses 2014 Gelegenheit, seine Arbeit einem größeren Publikum vorzustellen. Außerdem erhalten die Autoren der besten 3 Beiträge attraktive Geldpreise (jeweils 10000,6000 und $4000 €$ ).

Die Teilnahmebedingungen sowie das obligatorische Anmeldeformular können unter www.camlogfoundation.org/ awards heruntergeladen werden. Anmeldeschluss ist der 30. November 2013.

Nach einer Pressemitteilung der CAMLOG Foundation, CH-Basel

\title{
Unterschiedlichen Kundenbedürfnissen
}

\section{Rechnung tragen}

Experten gehen davon aus, dass bereits in wenigen Jahren sowohl im Praxis- als auch im Labormarkt signifikante Umsatzanteile durch digitale Prothetik und andere technisch basierte Dienstleistungen erzielt werden. Auf der anderen Seite steigen die Anforderungen im Bereich der Verbrauchsmaterialien hinsichtlich Materialvielfalt, Preiswürdigkeit und Service permanent an. Diesen Anforderungen will der Geschäftsbereich Heraeus Dental des Hanauer Edelmetall- und Technologiekonzerns Heraeus mit ihren neu gebildeten Divisionen „Digitale Services“ und „Dentale Materialien“ frühzeitig Rechnung tragen. Insbesondere im Bereich der Digitalen Services hat man sich ambitionierte Ziele gesetzt und ein Team von Experten zusammengezogen, die den Bereich zügig ausbauen sollen.

Auch die Marktbearbeitung der beiden Segmente Dentale Materialien und Digitale Services erfolgt in Zukunft getrennt. Damit erhöht das Unternehmen seine
Kundennähe noch einmal deutlich und schärft seine Beratungskompetenz. „Sowohl der Zahnarzt als auch der Laborinhaber brauchen einen unternehmerisch denkenden Partner, der ihm einerseits hilft, das Standardgeschäft möglichst effizient abzuwickeln, der ihn andererseits aber auch sicher in die neuen Geschäfte begleitet. Heraeus ist selbst ein inhaberorientiertes Unternehmen. Mit unserer klaren Fokussierung auf die beiden Bereiche Dentalmaterialien und Digitale Services sind wir in beiden Feldern für unsere Kunden der kompetente Partner, um die Zukunft zu gestalten“ ist Martin Haase, CEO von Heraeus Dental, überzeugt.

Die Neuausrichtung des Unternehmens auf die veränderten Marktbedürfnisse erfolgt ab sofort, die Veränderungen im Bereich werden aber noch in das Jahr 2013 hineinreichen.

\footnotetext{
Nach einer Pressemitteilung der Heraeus Kulzer GmbH, Hanau Internet: www.heraeus-dental.com
}

\section{Nachhaltiges}

\section{Engagement für die Mundgesundheit}

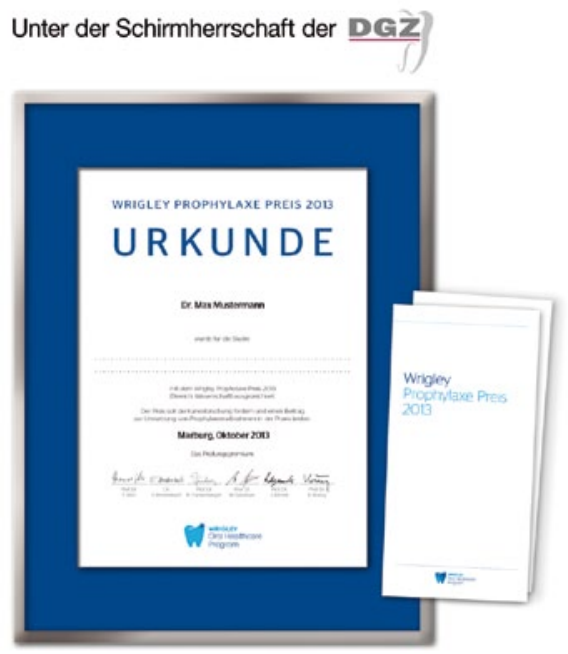

Alle, die sich in Forschung und Praxis mit der Prävention oraler Erkrankungen oder den Zusammenhängen zwischen Mund- und Allgemeingesundheit befassen, sind zur Bewerbung um den Wrigley Prophylaxe Preis 2013 aufgerufen. Dazu lädt die Gesundheitsinitiative Wrigley Oral Healthcare Program (WOHP) als Stifter des Preises ein. Die in zahnmedizinischen Kreisen angesehene Auszeichnung steht unter der Schirmherrschaft der Deutschen Gesellschaft für Zahnerhaltung (DGZ). Sie ist mit insgesamt $10000 €$ dotiert. Bewerbungen sind bis zum 1 . März 2013 willkommen. Verliehen wird die von der Wrigley GmbH Deutschland finanzierte Auszeichnung für neue Erkenntnisse in der Forschung und für die Umsetzung zahnmedizinischer Prävention in der Praxis, im öffentlichen Gesundheitswesen und in der Gruppenprophylaxe. Neben Praktikern und Zahnmedizinern sind auch Bewerbungen aus anderen naturwissenschaftlichen Fakultäten willkommen. Die feierliche Preisverleihung findet auf der DGZ-Jahrestagung vom 11.-12. Oktober 2013 in Marburg statt. Teilnahmebedingungen mit weiteren Informationen zu dem von einer unabhängigen Expertenjury geleiteten Auswahlverfahren sind unter www. wrigley-dental.de oder bei kommed Dr. Bethcke, Fax: 089/33036403, info@kommed-bethcke.de erhältlich.

Nach einer Pressemitteilung der

Wrigley Oral Healthcare 


\section{Die wissenschaftliche Herausforderung angenommen}

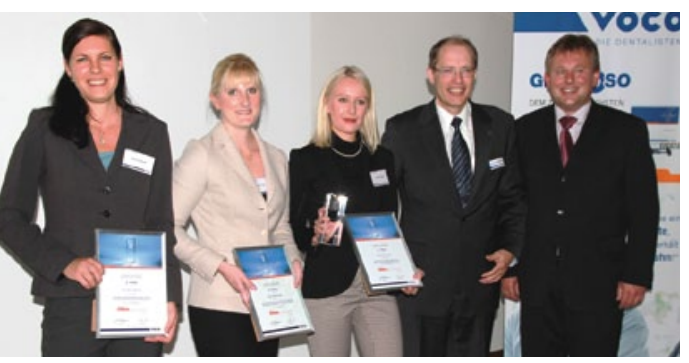

Die Preisträger der Voco Dental Challenge 2012: die Drittplatzierte Mona Seyfried, die Zweitplatzierte Sabine Kragt und die Erstplatzierte Simone Dudda (von links). Sie erhielten von VOCO-Geschäftsführer Manfred Thomas Plaumann (2.v.r.) und Dr. Martin Danebrock (Leiter Wissenschaftlicher Service, rechts) die Siegerurkunden sowie die Preisgelder.

Zum 10. Mal hat VOCO zur Dental Challenge im September nach Cuxhaven eingeladen. Elf Finalisten hatten die Chance, bei der Jubiläumsveranstaltung den begehrten Wertbewerb zu gewinnen. Den 3 Erstplatzierten winken neben der wissenschaftlichen Anerkennung ihrer Forschungen auch
Geldpreise und Publikationszuschüsse. Nicht nur dass ehemalige Gewinner als Gäste diesmal teilnehmen konnten, auch die Jury bestand aus 3 ehemaligen Preisträgern: OA PD Dr. Sebastian Hahnel (Regensburg), der Sieger von 2009, sowie OÄ Dr. Anne-Katrin Lührs (Hannover) und Dr. Arzu Tuna (Zahnärztin in eigener Praxis in Attendorn), die beide 2006 zu den Preisträgern zählten. Die Fachpresse war ebenfalls wieder dabei und diskutierte in den Pausen angeregt über die möglichen Preisträger. Die spätere Gewinnerin lag auch bei uns ganz weit vorn in der Bewertung.

Die Juroren gaben nach eingehenden Beratungen dann folgende 3 Preisträger bekannt: Simone Dudda (22), ZahnmedizinStudentin im 9. Semester an der Universität Marburg, belegte den 1. Platz mit einer spektakulären Präsentation zu einer interessanten Erfindung. Im Rahmen ihrer Arbeit „Optimierung der Kompositverarbeitung zur Reduktion schrumpfungsbedingter Spannungen“" entwickelte sie eine neue elektronisch gesteuerte Licht-Polymerisationstechnik, mit der sich Spannungen beim Legen von Komposit-Füllungen signifikant reduzieren lassen. Sabine Kragt (28), Assistenzzahnärztin in Moers und Doktorandin der Zahnmedizin in der Abteilung für Zahnerhaltung und Präventive Zahnheilkunde an der Universität WittenHerdecke, qualifizierte sich für den 2. Platz mit ihrer Arbeit zur „Remineralisation von humanem Dentin nach Applikation von Cerivtec $^{\circledR}$ und Bifluorid $10^{\circledR}$ - eine in-situStudie“. Mona Seyfried (29), Chemikerin und wissenschaftliche Mitarbeiterin am Fraunhofer Institut für Silicatforschung, errang den 3. Platz mit ihrer Arbeit „Neuartige selbstätzende Adhäsivsysteme auf Basis von Sulfonsäure-funktionalisierten Ormocer ${ }^{\circledR}$ en“.

Die Jubiläumsveranstaltung war auch die letzte in den alten Räumen im Hauptgebäude. VOCO erweitert für Forschung und Schulung, und wird zur 11. Challenge in das neue Zentrum einladen können. Gi/ZWR

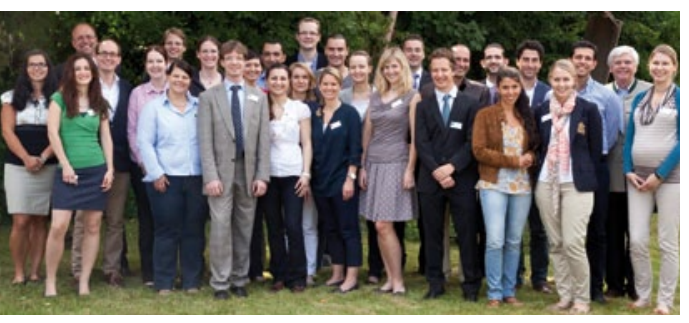

Äußerst zufrieden blicken Prof. Ralph G. Luthardt, Ärztlicher Direktor der Klinik für Zahnärztliche Prothetik am Universitätsklinikum Ulm, und sein Team auf die erfolgreich abgeschlossene 1 . Nachwuchsakademie Zahnmedizin zurück, die am Ulmer Institut für Lasertechnologien in der Medizin und Messtechnik (ILM) stattfand. „Wir sind mit dieser Prämierenveranstaltung unserem langfristigen Ziel, die Zahnmedizin in der Forschung zu stärken, näher gekommen, indem wir den wissenschaftlichen Nachwuchs konsequent und nachhaltig unterstützen“, so Prof. Luthardt zu Sinn und Zweck der Nachwuchsakademie.

Über insgesamt 5 Tage vermittelten renommierte Professoren nicht nur aus dem Bereich der Zahnmedizin theoretisches Wissen zu Themen wie Stammzellforschung und Publikationsstrategien. Aber auch praktische Hilfestellungen kamen nicht zu kurz: So bekamen die insgesamt 20 ausgewählten Nachwuchswissenschaftler in ausgedehnten Workshops zum Beispiel Hilfe und Tipps zur konkreten Ausgestaltung von Vollanträgen an die Deutsche Forschungsgemeinschaft (DFG). Dr. Frank Wissing, Programmdirektor bei der DFG, beantwortete zudem viele Fragen zur Vorgehensweise im Falle einer Förderung.

„Dieses Wissen findet sich in keinem Lehrbuch, hier konnten unsere Teilnehmer in einem großen Maße von den Mentoren profitieren“, verdeutlichen Dr. Katharina Kuhn und Stephanie Rupf aus der Klinik für Zahnärztliche Prothetik. Beide kümmerten sich um die Organisation der Nachwuchsakademie. Sie berichten, dass im Vorfeld insgesamt 65 Bewerbungen zur Teilnahme eingegangen waren. „Diese gute Resonanz ist ein sicheres Indiz für den großen Bedarf in diesem Bereich“, unterstreicht Dr. Kuhn und ergänzt: „Jeder Bewerber musste ein Forschungsthema skizzieren. Schnell wurde dabei deutlich, wie beeindruckend vielfältig die Interessenschwerpunkte von jungen Nachwuchswissenschaftlern sind.“

Die nächsten Schritte stehen schon fest: Bis zum 6. Januar 2013 haben die 12 Teilnehmerinnen und 8 Teilnehmer nun Zeit, ihre fertig ausgearbeiteten Forschungsanträge bei der DFG einzureichen. „Etwa die Hälfte dieser Anträge wird erfahrungsgemäß bewilligt“, erläutert Prof. Luthardt. „In der sich anschließenden rund 1-jährigen Vorarbeitsphase stehen dann jeweils 50000,- Euro zur Verfügung, um das Forschungsprojekt weiter ausarbeiten zu können“, so der Ärztliche Direktor. Im Sommer des kommenden Jahres ist die Klinik für Zahnärztliche Prothetik dann wiederum Gastgeber.

\footnotetext{
Nach einer Presseinformation der Presse- und Öffentlichkeitsarbeit Universitätsklinikum Ulm Jörg Portius

E-Mail: joerg.portius@uniklinik-ulm.de
} 